\title{
Impact of a shortened surveillance interval on hepatocellular carcinoma survival
}

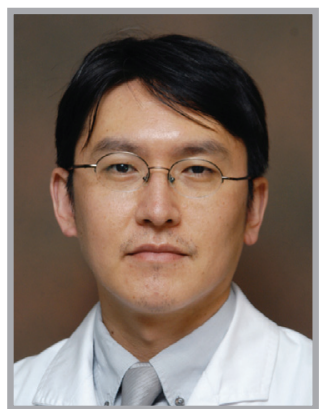

Do Young Kim

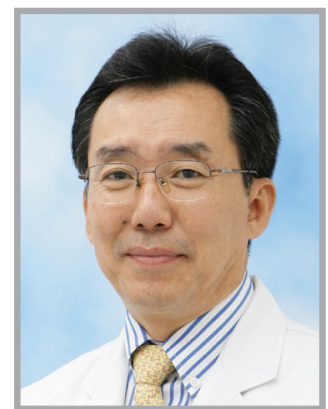

Kwang-Hyub Han*1

\author{
“...the superiority of a \\ 6-month interval compared \\ with a 12-month interval was \\ confirmed both in hepatitis $C$ \\ and $B$ virus-related chronic \\ liver diseases.”
}

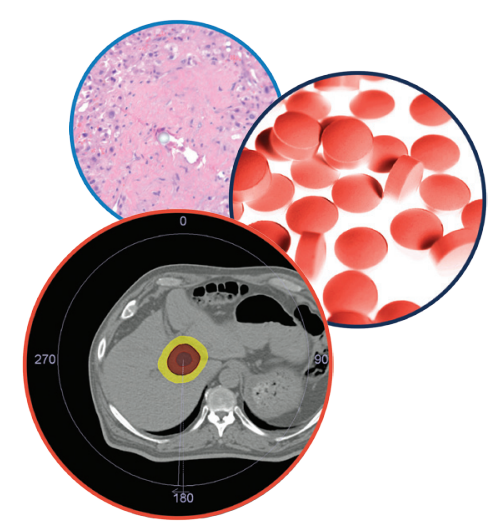

The surveillance of hepatocellular carcinoma (HCC), which has been a standard of care in the management of patients with chronic liver diseases, greatly contributed to the early detection of liver cancers amenable to curative treatment. Nonetheless, the current strategy for HCC surveillance does not seem to be ideal and the problems may mostly arise from the incompleteness of surveillance tools. As revealed by a study using the cohort of the HALT-C trial, the most common reason for identifying HCC at a late stage was an absence of detection despite adherence to surveillance, which clearly implies that better surveillance tools are required [1]. Ultrasonography (US) is a noninvasive, safe and easy-to-apply method in HCC surveillance, showing a sensitivity of $65-80 \%$ [2]. However, the performance characteristics of US becomes poor in obese patients or nodular cirrhotic livers. The role of adding serum $\alpha$-fetoprotein (AFP) measurement complementary to US is still in debate between eastern and western countries. Unfortunately, a marked progress has not yet been made in terms of development or discovery of novel noninvasive imaging (or a serum biomarker) that could outperform US or AFP.
Looking at the recommendation by American Association for the Study of Liver Diseases, the proposed interval of 6-12 months for HCC surveillance is based on the tumor doubling time $[3,4]$. Although initial studies of small sample size reported that there was no difference in the rate of early HCC detection or survival between 6 and 12 months [5,6], in an Italian study, the median survival of patients with a 6 -month interval was significantly longer than those with a 12 -month interval, after correction of lead-time bias [7]. Furthermore, in a 15 -year prospective Korean study, the corrected 5-year survival of patients, whose surveillance interval was less than 6 months, was significantly higher compared with those with an interval of more than 6 months [8]. Accordingly, the superiority of a 6-month interval compared with a 12-month interval was confirmed both in hepatitis $\mathrm{C}$ and $\mathrm{B}$ virus-related chronic liver diseases. Based on such recent data, a 6-month interval should be preferred over 12 months in clinical practice.

A basic concept regarding HCC surveillance is that determination of interval should be made according to the tumor growth rate, not by the degree of tumor “...the current evidence suggests that shortening of

ultrasonographic surveillance to 3-4 months does not impact on survival of hepatocellular carcinoma patients." 
"The remaining issue is whether or not shortening the surveillance interval could be beneficial when both ultrasonography and $\alpha$-fetoprotein are utilized." development risk [3]. However, as stated above, the failure to detect lesions appropriately is the most frequently encountered situation in patients diagnosed with HCC beyond early stage, despite surveillance. Hence, the issue of shortening the HCC surveillance interval may come from the attempt to make up for the limitation of US itself or operational imperfection. To address this issue, a multicenter European study has been conducted, where 1278 cirrhotic patients were randomized to US every 6 or 3 months [9]. HCC was diagnosed in 123 patients $(9.6 \%)$, and there was no difference in HCC incidence between the two groups. The prevalence of $\mathrm{HCC}<3 \mathrm{~cm}$, which was the main end point of that study, was also not different, although the incidence of small lesions $(\leq 10 \mathrm{~mm})$ was higher in the 3-month group compared with the 6-month group. Finally, the cumulative 5-year survival was found to be similar between the randomized groups. The result of this trial could make clinicians believe that shortening surveillance interval from 6 to 3 months is useless in terms of small HCC detection or survival increase, at least in western countries.

A recently published Taiwanese study also tried to investigate whether a shortened surveillance interval could improve patient survival [10]. A total of 744 patients with chronic hepatitis $\mathrm{C}$ or $\mathrm{B}$ virus infection were prospectively enrolled and randomized to US every 4 or 12 months. Although there was no difference in cumulative 3-year HCC incidence between the two groups, the 4-month group had significantly more patients with tumor $\leq 2 \mathrm{~cm}$ compared with the 12-month group. However, contrary to the results from aforementioned Korean or Italian studies, the 4-year overall survival was not different between two groups. The reason why patients in the 4-month group did not achieve a better survival than those in the 12-month group could be explained by nonadherence of early stage patients to curative treatment, such as resection or ablation, owing to various reasons including old age or comorbidities. The survival of the 4-month group tended to be higher compared with the 12-month group in early follow-up period; however, the survival curves in the two groups merged in later periods. Obviously, the effectiveness of surveillance strategy has led to the decrease of mortality due to HCC. Thus, the current evidence suggests that shortening of US surveillance to 3-4 months does not impact on survival of HCC patients.

Should we therefore be satisfied with a surveillance interval of 6 months using US? Is there no room for increasing surveillance outcome by modifying surveillance strategy? Interestingly, the two prospective European and Taiwanese studies adopted only US for HCC surveillance. However, although western liver society does not accept the role of serum AFP measurement in HCC surveillance owing to its low sensitivity and specificity, it is a routine practice to combine US and AFP in many eastern and even some western countries. We recognize that 6 months is the most appropriate interval if HCC surveillance is performed with only US, based on the accumulated evidence.

The remaining issue is whether or not shortening the surveillance interval could be beneficial when both US and AFP are utilized. It is helpful for us to remind that most HCCs are detected at an early stage in Japan, where a shortened surveillance interval and combination of US and several tumor markers such as serum AFP and PIVKA-II are already the standard of care. Although the cost-effectiveness of surveillance is one of the most important factors to consider, the improvement of sensitivity to diagnose HCC at an early stage is also an important issue.

Financial \& competing interests disclosure

The authors have no relevant affiliations or financial involvement with any organization or entity with a financial interest in or financial conflict with the subject matter or materials discussed in the manuscript. This includes employment, consultancies, honoraria, stock ownership or options, expert testimony, grants or patents received or pending, or royalties.

No writing assistance was utilized in the production of this manuscript.

\section{References}

1 Singal A, Nehra M, Adams-Huet B et al. Detection of hepatocellular carcinoma at advanced stages among patients in the HALT-CT trial: where did surveillance fail? Am. J. Gastroenterol. 108, 425-432 (2013).

2 Bolondi L, Sofia S, Siringo S et al. Surveillance programme of cirrhotic patients for early diagnosis and treatment of hepatocellular carcinoma: a cost-effectiveness analysis. Gut 48, 251-259 (2001)

3 Bruix J, Sherman M; Practice guidelines committee, American Association for the Study of Liver Diseases. Management of hepatocellular carcinoma. Hepatology 42 , 1208-1236 (2005).
4 Bruix J, Sherman M; American Association for the Study of Liver Diseases. Management of hepatocellular carcinoma: an update. Hepatology 53, 1020-1022 (2011).

5 Trevisani F, De NS, Rapaccini G et al. Semiannual and annual surveillance of cirrhotic patients for hepatocellular carcinoma: effects on cancer stage and patient 
survival (Italian experience). Am. J. Gastroenterol. 97, 734-744 (2002).

6 Santagostino E, Colombo M, Rivi M et al. A 6-month versus a 12 -month surveillance for hepatocellular carcinoma in 559 hemophiliacs infected with the hepatitis C virus. Blood 102, 78-82 (2003).

7 Santi V, Trevisani F, Gramenzi A et al. Semiannual surveillance is superior to annual surveillance for the detection of early hepatocellular carcinoma and patient survival. J. Hepatol. 53, 291-297 (2010).

8 Han KH, Kim do Y, Park JY et al. Survival of hepatocellular carcinoma patients may be improved in surveillance interval not more than 6 months compared with more than 6 months: a 15 -year prospective study. J. Clin. Gastroenterol. 47, 538-544 (2013).

9 Trinchet JC, Chaffaut C, Bourcier V et al. Ultrasonographic surveillance of hepatocellular carcinoma in cirrhosis: a randomized trial comparing 3 - and 6-month periodicities. Hepatology 54 , 1987-1997 (2011)

10 Wang JH, Chang KC, Kee KM et al. Hepatocellular carcinoma surveillance at 4- vs. 12-month intervals for patients with chronic viral hepatitis: a randomized study in community. Am. J. Gastroenterol. 108, 416-424 (2013). 